\title{
High Luminosity Spin-Polarized Target for the SpinQuest Experiment
}

New Perspectives 2019

Joshua Hoskins

University of Virginia

Polarized Target Group

This manuscript has been authored by Fermi Research Alliance, LLC under Contract No. DE-AC02-07CH11359 with the U.S. Department of Energy, Office of Science, Office of High Energy Physics. 


\section{Overview}

- SpinQuest experiment at Fermilab

- Spin Polarized Solid Targets 


\section{Proton's Spin Structure}

Central challenge of nuclear physics: to disentangle constituents and spin structure of the nucleon.

Since the proposal of the parton model our understanding of the nuclear constituents, quarks and gluons, has grown immensely.

The EMC experiment, by the discovery that the contribution of the spins of the quark and anti-quark to the proton's spin was $12 \pm 17 \%$, sparked a multi-decade program with the purpose of understanding the spin structure of the proton.

$$
\frac{1}{2}=\frac{1}{2} \Delta \Sigma+L_{q}+L_{\bar{q}}+\Delta G+L_{g}
$$

Tremendous progress has been made in understanding how the nucleon spin is made up from its quark and gluon constituents.

The current best estimate of the contribution of the quark and anti-quark spin to the proton's spin is only $\sim 30 \%$.

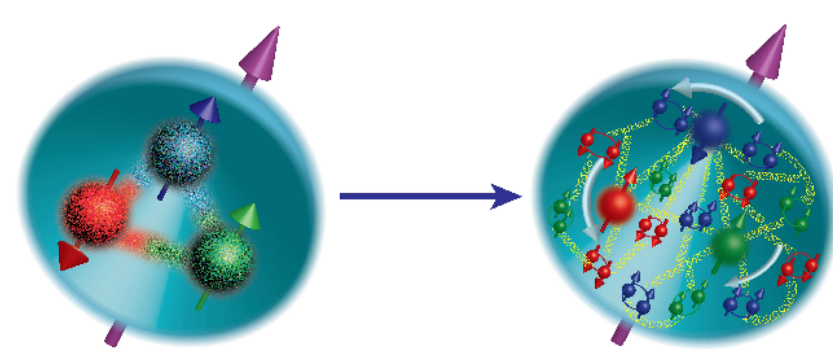

CT10.00 PDFs

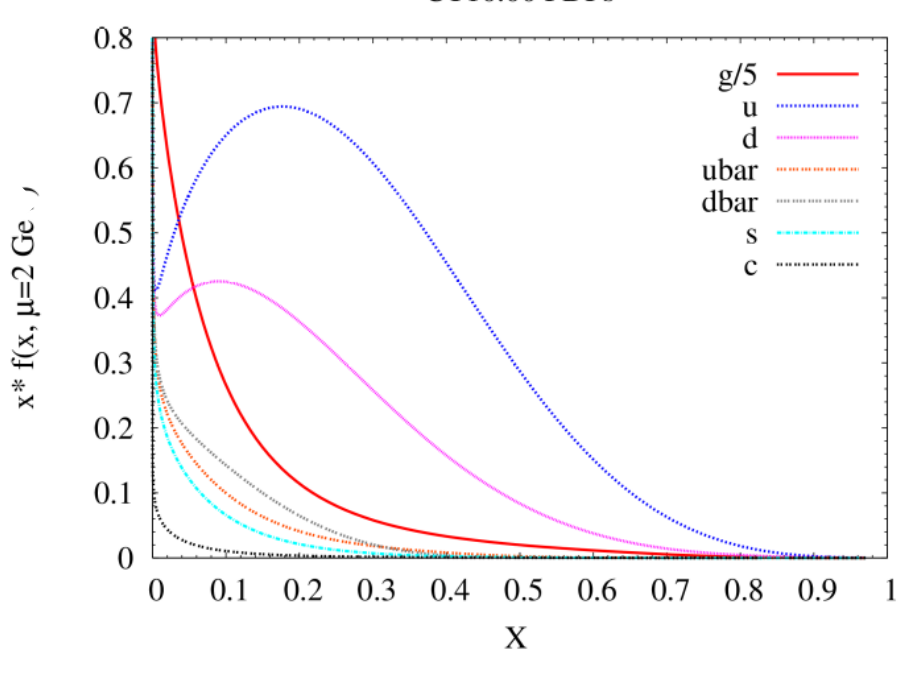




\section{Spin Physics and E1039 (SpinQuest)}

SpinQuest proposes direct measurement of the sea-quark Sivers function, using Drell-Yan productions from unpolarized $120 \mathrm{GeV}$ proton beam on a transversely polarized proton (neutron) target.

Sivers function - one of eight transverse momentum distribution functions (TMD), describing the correlation of the transverse momentum of an unpolarized parton with the spin of a polarized nucleon.

Interest in the contributions of the orbital motion of the sea quarks $\rightarrow$ data are severely lacking.

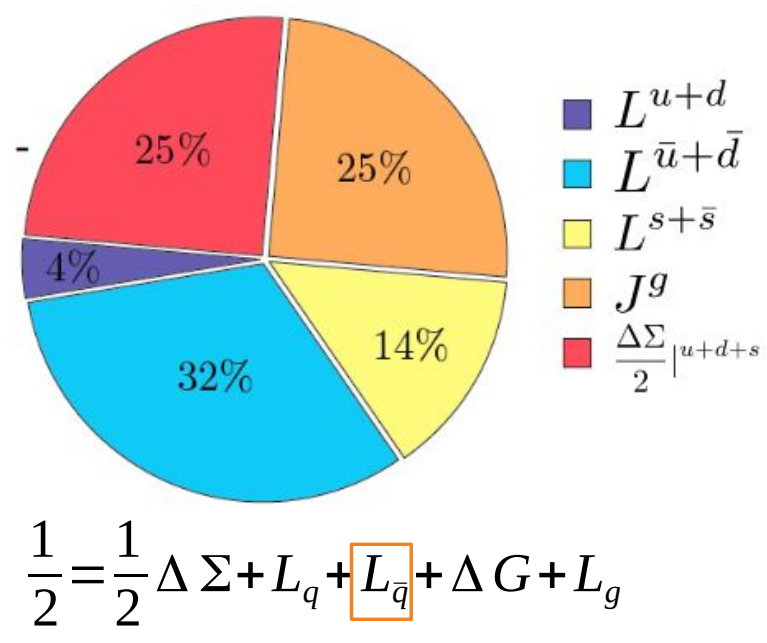

$\rightarrow$ HERMES, COMPASS, Jefferson Lab have provided measurements of the Sivers Function via semi-inclusive deep inelastic scattering (SIDIS); Sivers function convoluted with fragmentation function.

1) Fundamental prediction of $Q C D \rightarrow$ Sivers function equal in magnitude and opposite $A_{N}^{D Y} \propto \frac{\sum_{q} e_{q}^{2}\left[f_{1}^{q}\left(x_{1}\right) \cdot f_{1 T}^{\perp, \bar{q}}\left(x_{2}\right)+1 \leftarrow \rightarrow 2\right]}{\sum_{q} e_{q}^{2}\left[f_{1}^{q}\left(x_{1}\right) \cdot f_{1}^{\bar{q}}\left(x_{2}\right)+1 \leftarrow \rightarrow 2\right]}$

2) A non-vanishing Sivers function provides a strong indicator of non-zero sea-quark orbital momentum!

$f \frac{1}{1 T}\left(x, k_{T}\right)=\bigodot-\bigodot$ 
Polarized Target 


\section{Spin Polarized Solid Targets}

How do we obtain significant nucleon polarization?

The simplest way is via interaction with magnetic moment, $\mu$.

This is Zeeman splitting.

An ensemble of particles cooled and placed in an external B-field create $2 \mathrm{~J}+1$ energy levels

The vector polarization at thermal equilibrium is given to be:

$$
P_{T E}=\frac{e^{\mu B / k T}-e^{-\mu B / k T}}{e^{\mu B / k T}+e^{-\mu B / k T}}=\tanh \left(\frac{\mu B}{k T}\right)
$$

\section{Brute Force: High B-field, Low T}

- Simple and works for most any material.

- Requires high magnetic field

- Low temperatures $\rightarrow$ low luminosity

- Takes a long time to polarize.

... but there is a better way!
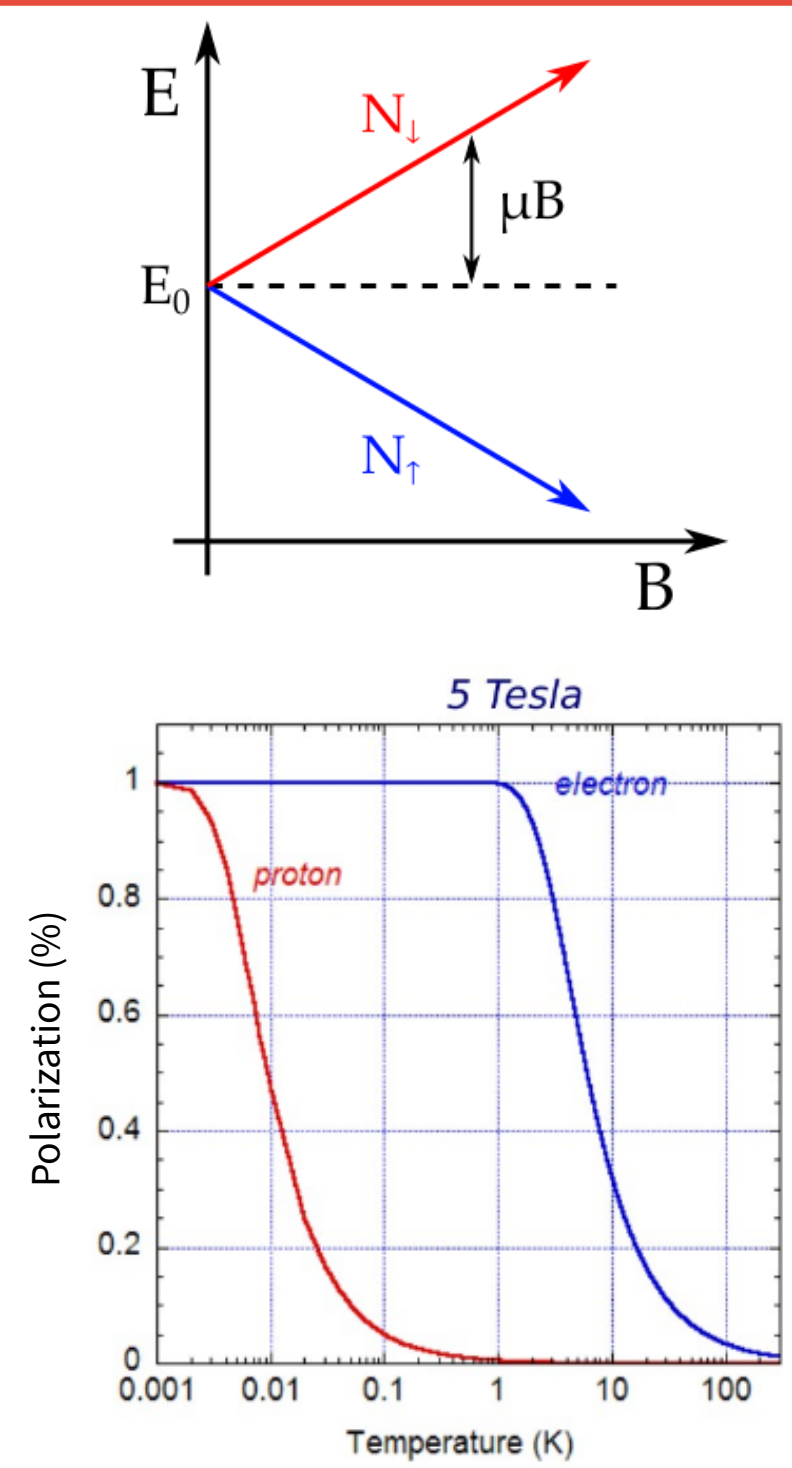


\section{Spin Polarized Solid Targets}

How do we obtain significant nucleon polarization?

The simplest way is via interaction with magnetic moment, $\mu$.

This is Zeeman splitting.

An ensemble of particles cooled and placed in an external B-field create $2 \mathrm{~J}+1$ energy levels

The vector polarization at thermal equilibrium is given to be:

$$
P_{T E}=\frac{e^{\mu B / k T}-e^{-\mu B / k T}}{e^{\mu B / k T}+e^{-\mu B / k T}}=\tanh \left(\frac{\mu B}{k T}\right)
$$

\section{Dynamic Nuclear Polarization: High B-field, Moderate T}

- Use doped target materials with paramagnetic centers.

- Centers are polarized by external magnetic field.

- Use microwave to transfer polarization to the nucleon via pumping.

- The disparity in relaxation times between the electron(ms) and the nucleon (minutes $\rightarrow$ hours) makes high spin polarization possible.
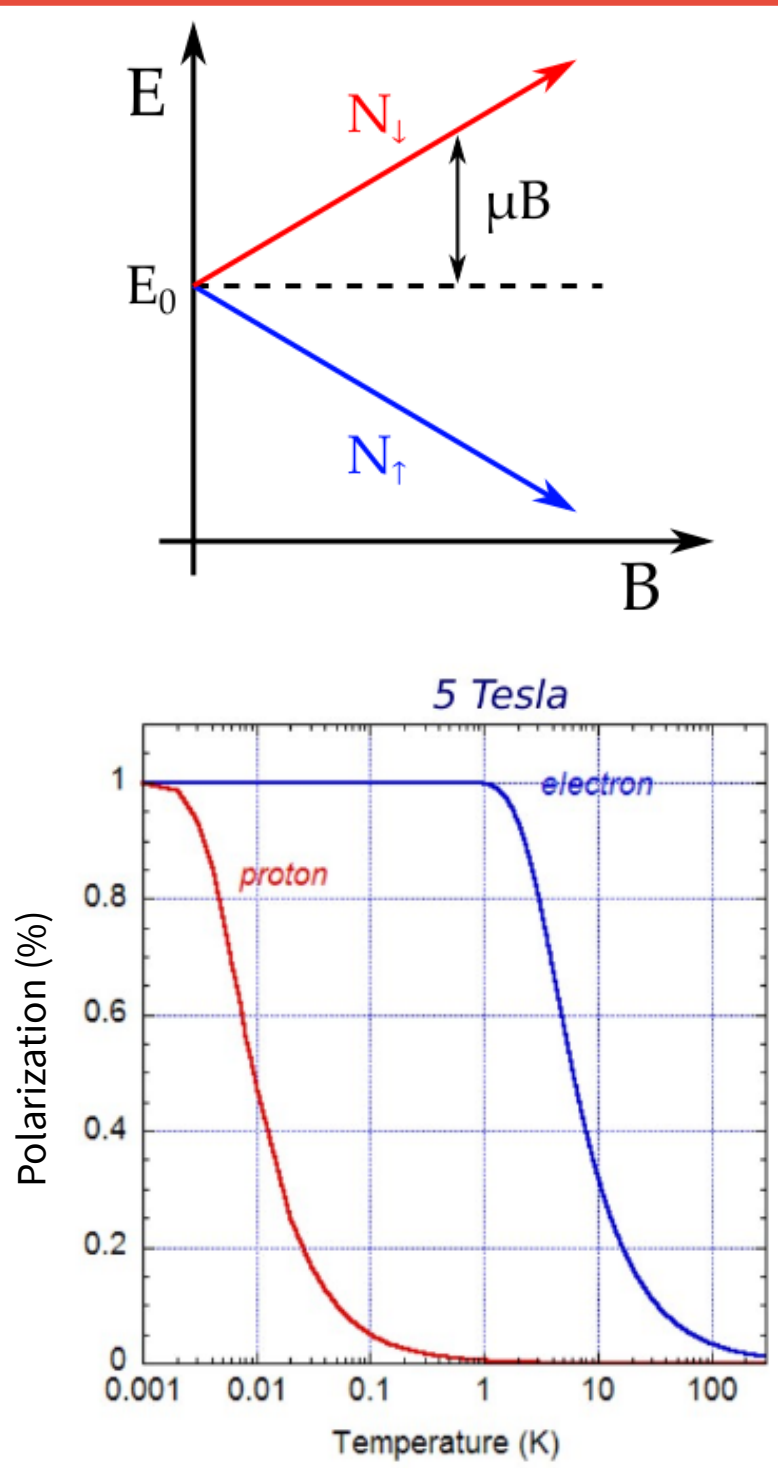


\section{Dynamic Nuclear Polarization}

For a system with of unpaired electrons in the target material, hyper-fine splitting gives four discrete energy levels.

$$
H=\mu_{e} B+\mu_{p} B+H_{s s}
$$

Applying an RF signal at the proper frequency we can drive the nucleons into a preferential state; the relatively short relaxation time of the electron versus the proton is crucial in pumping the polarization to a higher value.

The electrons spins are flipped using an RF-signal at the electron paramagnetic resonance frequency (EPR).

The proton spins are flipped using a RF-signal at the nuclear paramagnetic resonance frequency (NMR).

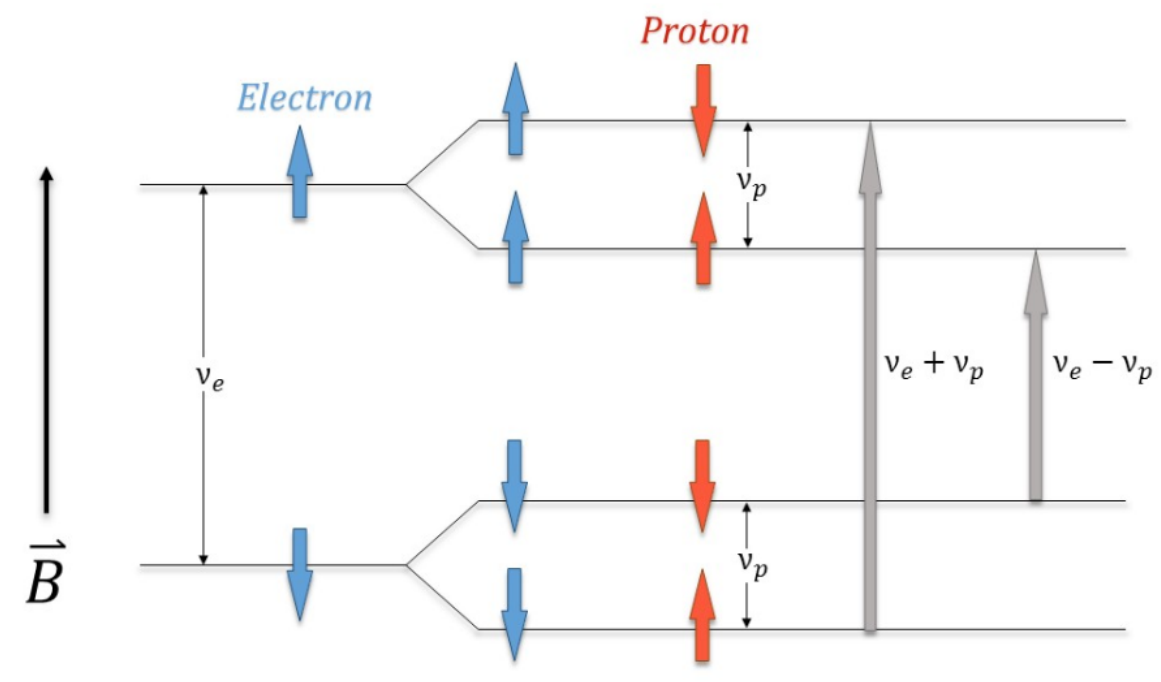

Positive or negative nucleon polarization can be achieved using the proper RF-signal frequency.

Spin flip is B-field independent and requires only a change in RF frequency! 


\section{Polarized Target Overview}

LANL-UVA Polarized Target: Designed for high intensity proton beam $\left(4 \times 10^{12}\right.$ proton $\left./ 4 \mathrm{sec}\right)$

- 5T superconducting split coil magnet.

- ${ }^{4} \mathrm{He}$ evaporation refrigerator (1W cooling).

- $140 \mathrm{GHz}$ microwave source.

- $14000 \mathrm{~m}^{3} / \mathrm{hr}$ pumping system.

- $8 \mathrm{~cm}$ long solid $\mathrm{NH}_{3} / \mathrm{ND}_{3}$ targets.

- Luminosity of $2 \times 10^{35} \mathrm{~cm}^{-2} \mathrm{~S}^{-1}$

- $d B / B<10^{-4}$ at 5 T over $8 \mathrm{~cm}$

\begin{tabular}{|l|l|c|c|c|}
\hline Material & Density & Dilution Factor & Packing Fraction & $<$ Polarization> \\
\hline $\mathrm{NH}_{3}$ & $0.867 \mathrm{~g} / \mathrm{cm}^{3}$ & 0.176 & 0.60 & $80 \%$ \\
\hline $\mathrm{ND}_{3}$ & $1.007 \mathrm{~g} / \mathrm{cm}^{3}$ & 0.300 & 0.60 & $32 \%$ \\
\hline
\end{tabular}

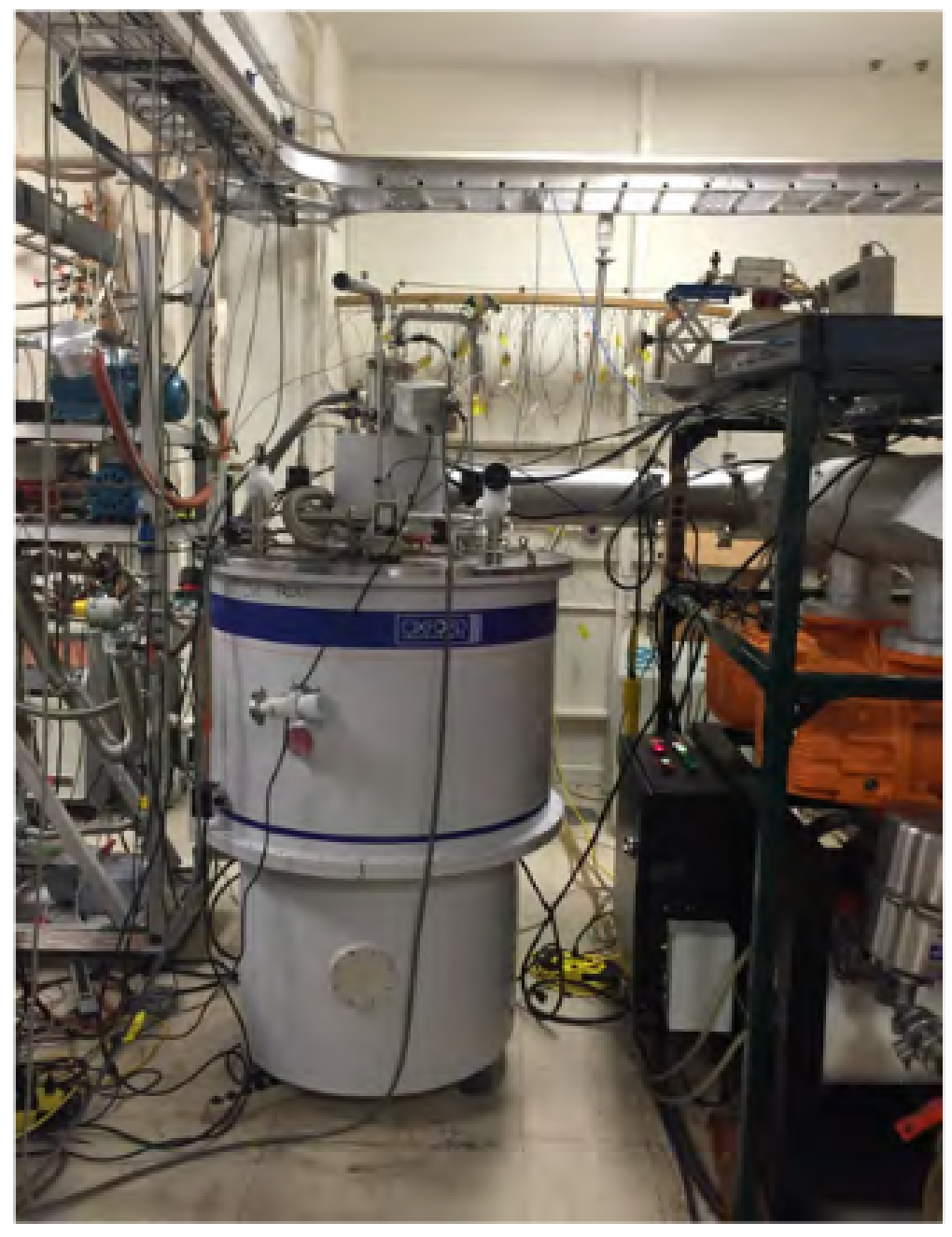




\section{Spin Polarized Solid Target}

The polarized target consists of three main systems:

- The microwave system which pumps the spin polarization of the target

- The NMR system which measures the target polarization.

- The cryogenics and pumping system which cools the solid target and magnet coils

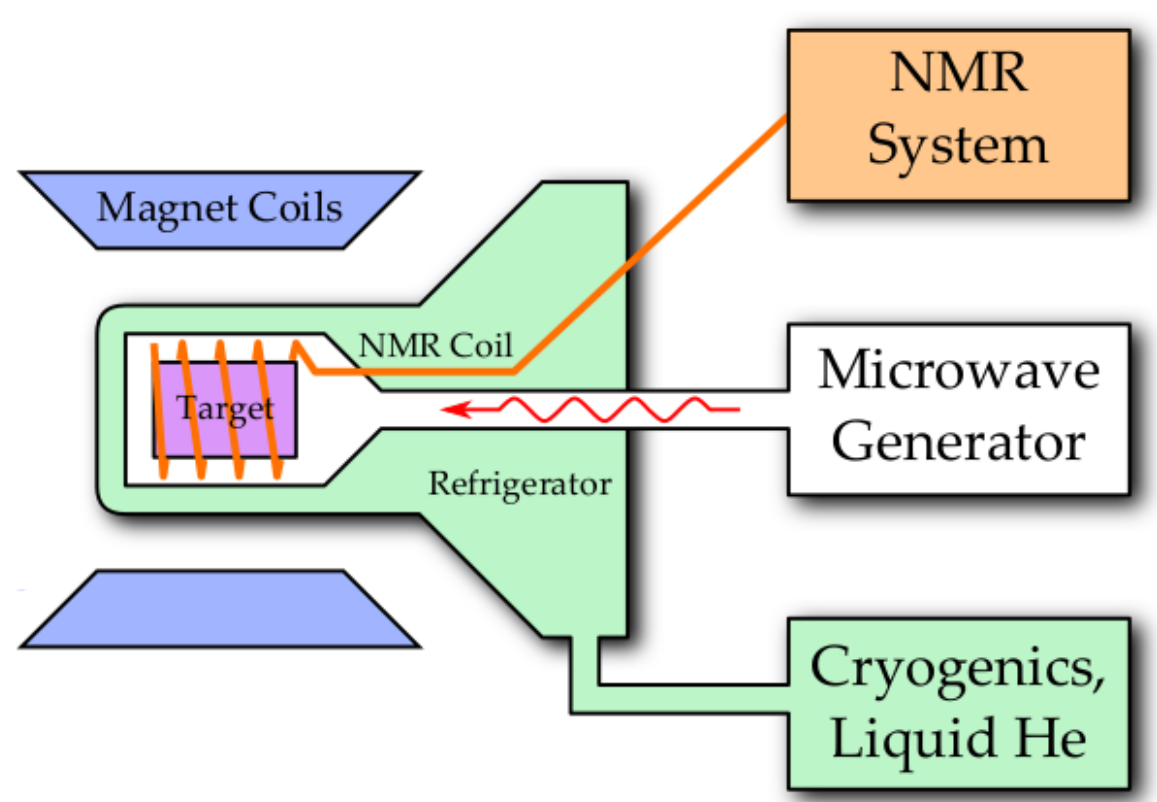




\section{Microwave System}

Microwaves used to drive dynamic polarization enhancement $\rightarrow$ Extended Interaction Oscillator(EIO)

The EIO is coupled to the target material cups via a wave-guide inserted into the target.

The wave-guide is terminated at a gold plated copper horn trained on the target material cups.

Driving frequency defined by ESP and NMR.

- ElO attenuated from $20 \mathrm{~W} \rightarrow 1 \mathrm{~W}$ scale.

- $140 \mathrm{GHz}$ ( $\mathrm{F} \rightarrow \mathrm{D}$ wave) microwaves

- Cavity adjustment via stepper motor allows for $1.5 \%$ frequency adjustment.

- Voltage adjustment allows for another $0.5 \%$ adjustment.
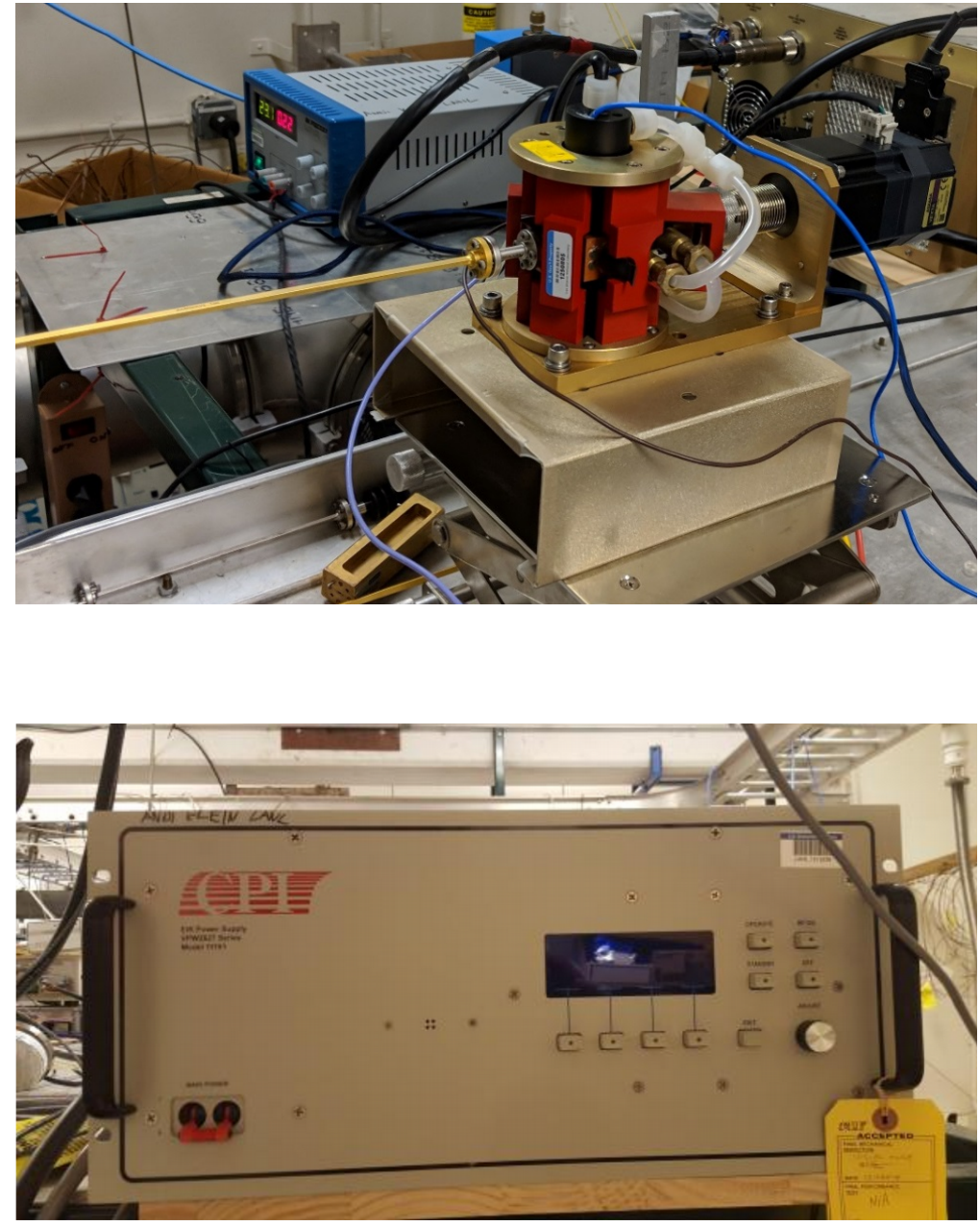


\section{Nuclear Magnetic Resonance Systems}

Polarization measured using nuclear magnetic resonance.

Each target cup contains an NMR coil which makes up part of an LRC tank circuit. Each spin flip (absorption/emission) changes effective coil impedance

The amount of energy is proportional to the polarization.

Circuit is tuned to the Larmor frequency of the sample (213 $\mathrm{MHz}$ at $5 \mathrm{~T}$ proton).

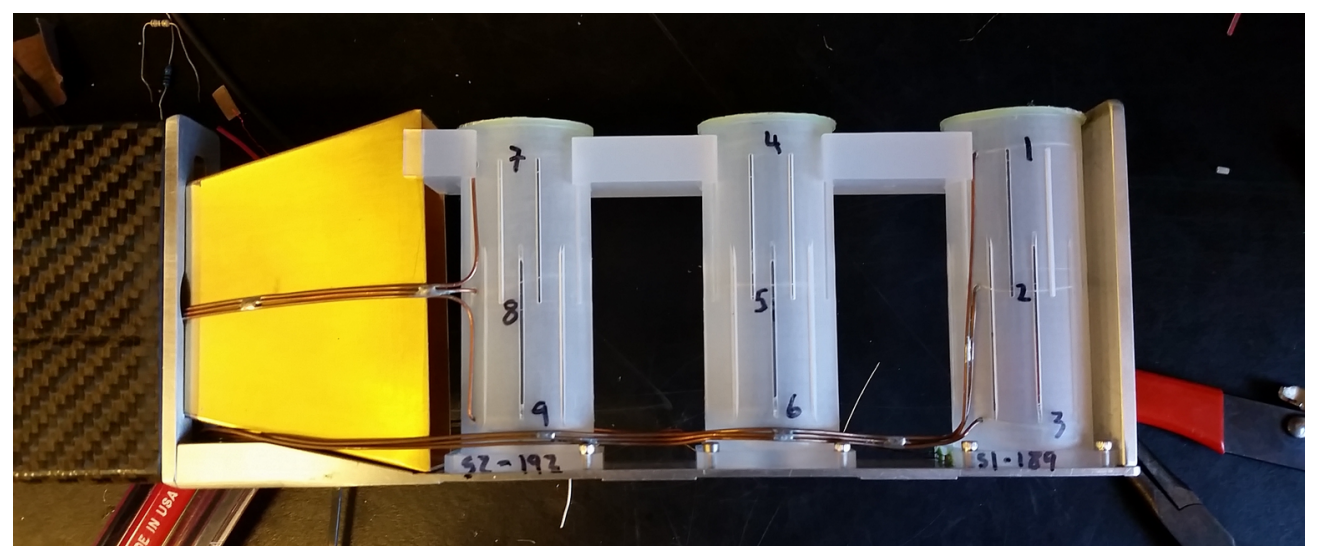

Liverpool Q-meter

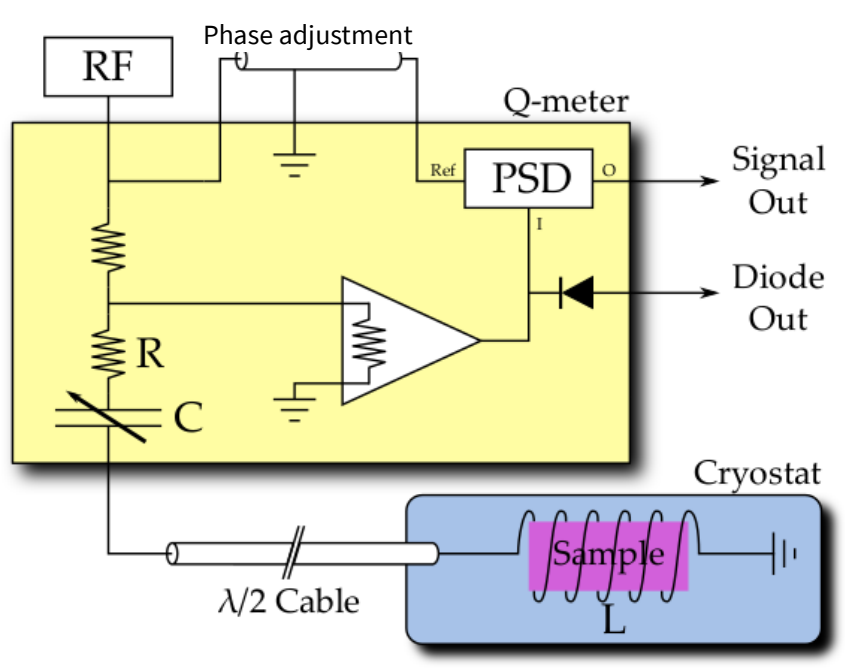

RF supplied as a frequency scan around resonance.

This produces a distinct characteristic curve - Q-curve even when no sample response is present.

This background is subtracted and the remaining signal area is proportional to the sample polarization. 


\section{Nuclear Magnetic Resonance Systems}

Polarization measured using nuclear magnetic resonance.

Each target cup contains an NMR coil which makes up part of an LRC tank circuit. Each spin flip (absorption/emission) changes effective coil impedance

The amount of energy is proportional to the polarization.

Circuit is tuned to the Larmor frequency of the sample (213 $\mathrm{MHz}$ at $5 \mathrm{~T}$ proton).
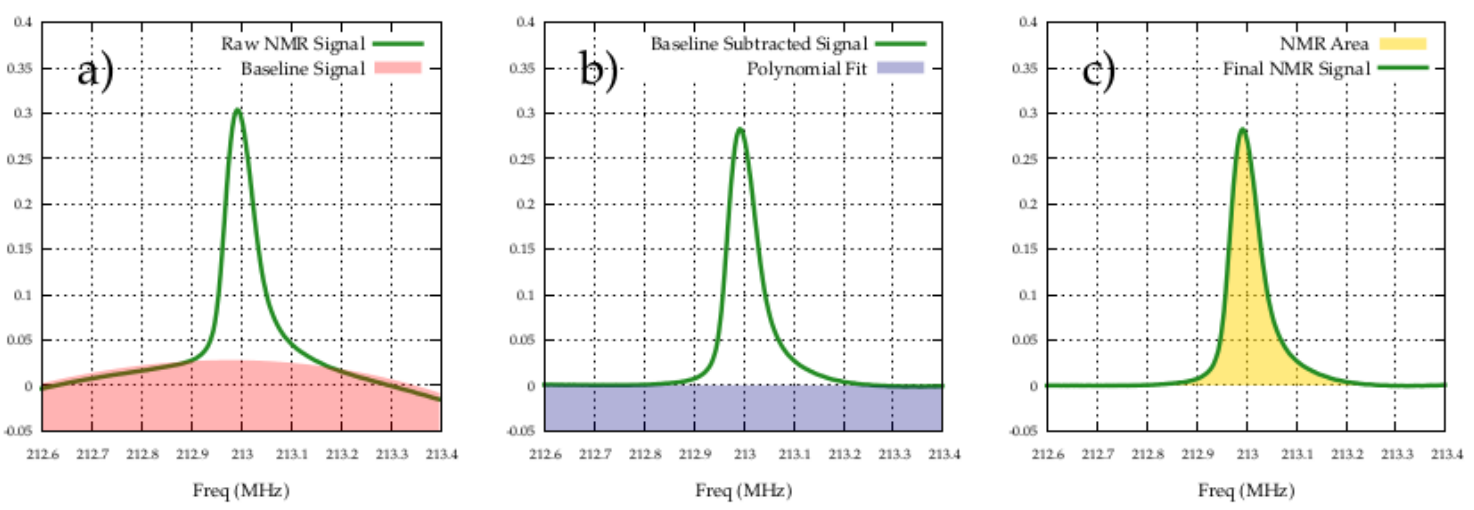

Liverpool Q-meter

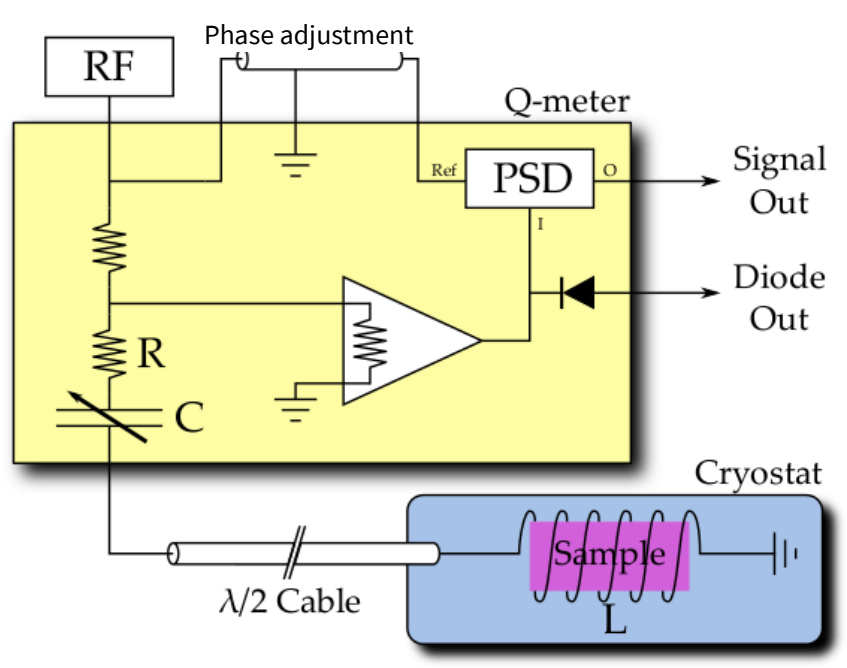

RF supplied as a frequency scan around resonance.

This produces a distinct characteristic curve - Q-curve even when no sample response is present.

This background is subtracted and the remaining signal area is proportional to the sample polarization. 


\section{Target Materials}

Choice of target material a balance between desire to have a pure proton target and the practicality of high radiation and DNP performance.

Materials should have potential for high polarization, maximal dilution factor, and be radiation hard.

Ammonia and deuterated ammonia are most attractive candidates at the moment.

Materials are doped with paramagnetic free-radicals by being irradiated at NIST.

Provides unpaired electron spins ( $99 \%$ at $1 \mathrm{~K} / 5 \mathrm{~T}$ ) that can be used in DNP to provide high polarization to the nucleon.

The maximum polarization reached with the proton(deuteron) target is $98 \%$ (48\%).

Successful material for DNP characterized by three measures:

1. Maximum polarization

2. Dilution factor

3. Resistance to ionizing radiation

$\begin{array}{llll} & \\ & & \\ & & \end{array}$




\section{Target Cooling and Evaporation Refrigerator}

The evaporation refrigerator uses ${ }^{4} \mathrm{He}$ to provide a cooling power of $1 \mathrm{~W}$ at $1 \mathrm{~K}$.

Cooling need to remove heat from the beam on the solid target - the refrigerator sits inside of the cryostat and the target insert slides into the refrigerator.

The refrigerator includes annealing plates for retrieving lost polarization in the target material due to beam heating.

Beam
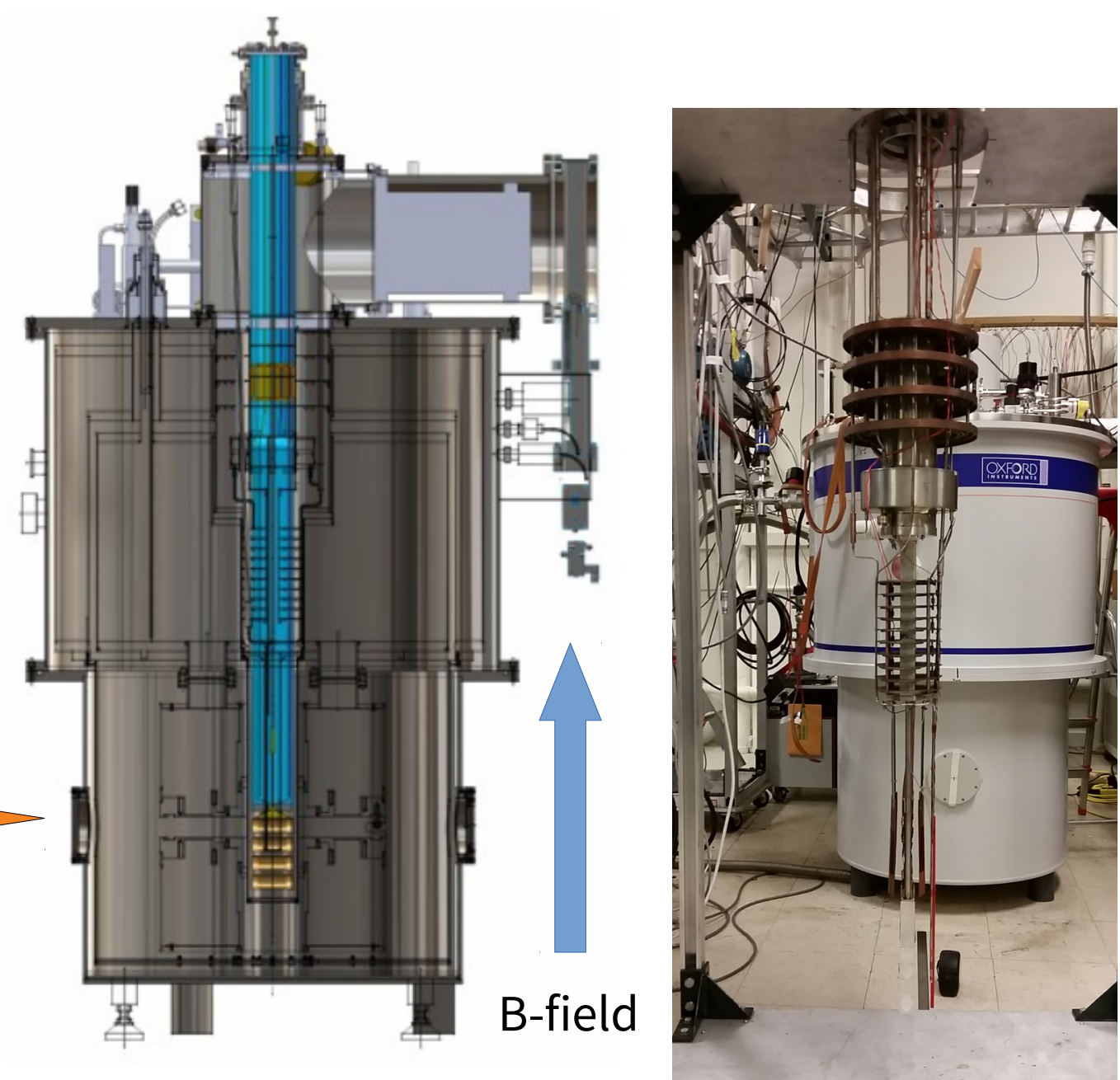


\section{Target Cooling and Evaporation Refrigerator}

The target ladder sits in the nose of the refrigerator cooled by a $1 \mathrm{~K}$ Helium bath.

Liquid helium at $4 \mathrm{~K}$ is drawn from a reservoir in the magnet through an insulated jumper and into the separator.

The separator is pumped on by and external pump and through evaporation the Helium temperature is lower to around 3K.

The Helium reaches the nose through either the run valve or the bypass valve.

Evaporated Helium from the nose convectively cools the refrigerator as it is pumped out

The insulating vacuum is $\sim 10^{-7}$ torr and liquid Nitrogen(77K) shield is used to prevent blackbody radiation loses to the outer walls of the cryostat (300K).
High powered pumps are needed to keep low pressure in the nose and deal with the high flow-rate.

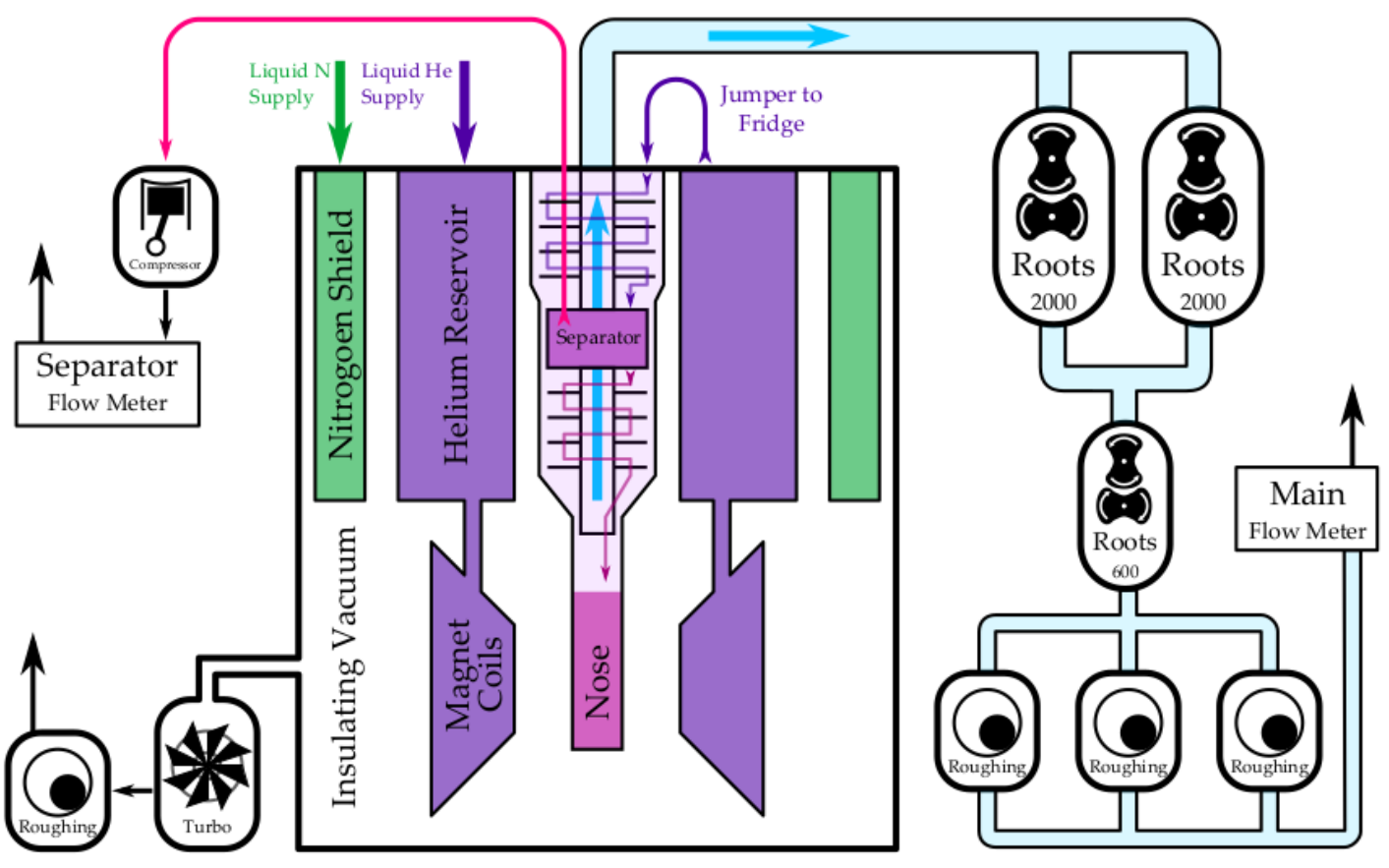

Run valve - runs through heat exchanger to further lower the Helium temperature.

Bypass valve - goes directly to the nose. 


\section{Effects of Beam Heating: Depolarization and Anneling}

As radiation dose from the beam continues, the DNP process becomes less efficient and the polarization will fall.

By heating the target material, we can temporarily restore the DNP process again. This heating is called annealing.

There is a limit to the lifetime however.
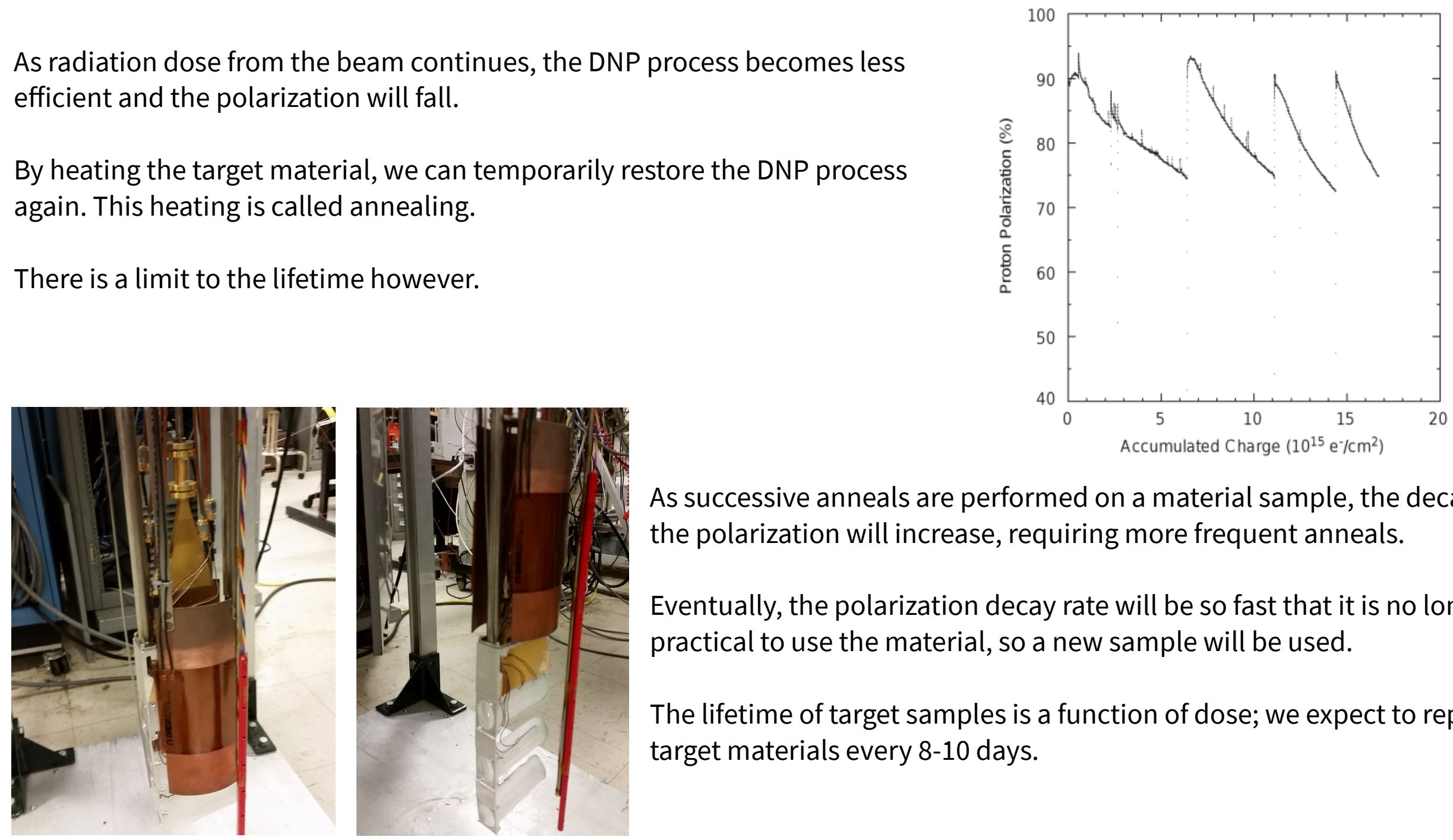

As successive anneals are performed on a material sample, the decay rate of the polarization will increase, requiring more frequent anneals.

Eventually, the polarization decay rate will be so fast that it is no longer practical to use the material, so a new sample will be used.

The lifetime of target samples is a function of dose; we expect to replace the target materials every 8-10 days. 


\section{Commissioning and Installation}

- Shielding, cryogenics, and infrastructure work in progress now at Fermilab

- Polarized target cooled down and tested, November 2018. Achieved $+90 \%$ polarization.

- Local testing of the polarized target done - most work is focused on optimizing subsystems and studying heat transfer due to beam on magnet coils.

- Target scheduled for installation/commissioning at Fermilab this summer.

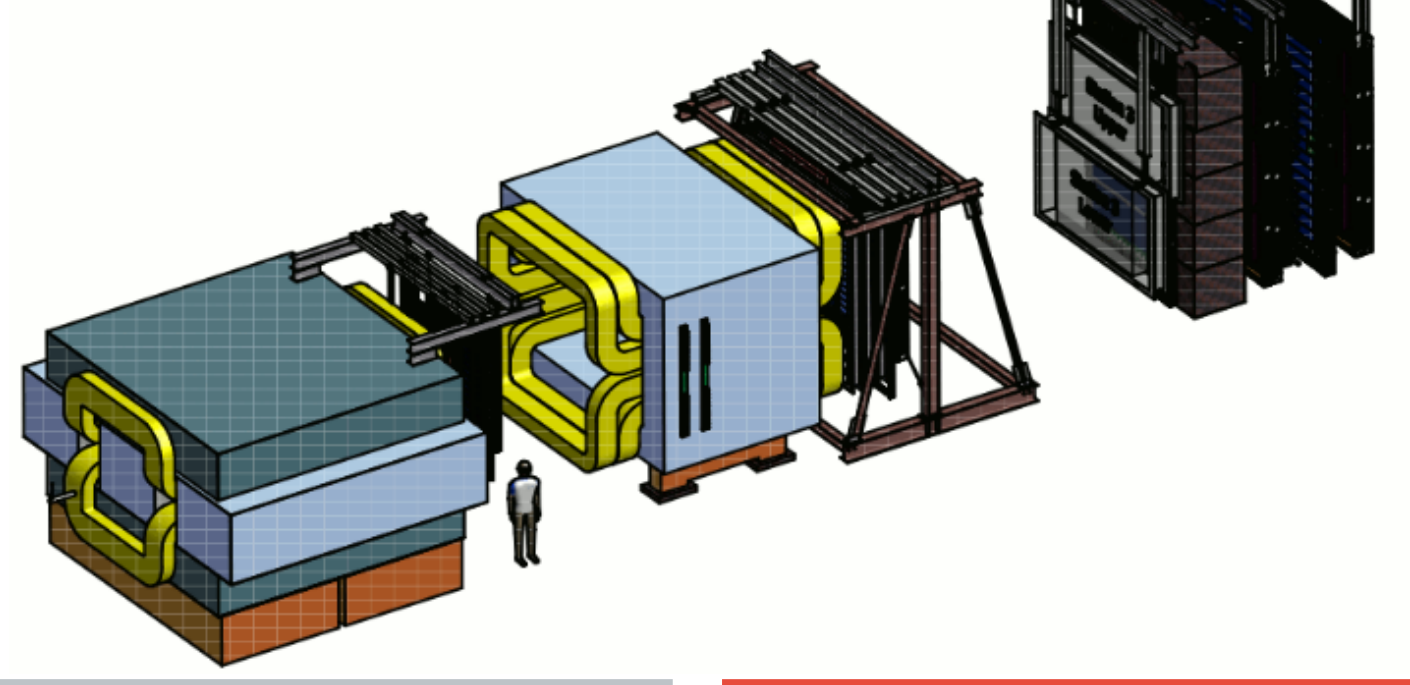




\section{Thank You!}

We are inviting collaborators to e1039!

Contact(s): Kun Liu (liuk@fnal.gov) - LANL

Dustin Keller (dustin@jlab.org) - UVA

Or feel free to talk to me during workshop

Learn more about E1039: $\quad$ https://spinquest.fnal.gov/ 


\section{Backup Slides}




\section{Spin Physics and E1039 (SpinQuest)}

SpinQuest purposes direct measurement sea-quark Sivers function using Drell-Yan productions from unpolarized $120 \mathrm{GeV}$ proton beam on a transversely polarized proton (neutron) target.

$$
e+p^{\uparrow} \rightarrow e^{\prime} \pi X
$$

Polarized Semi-Inclusive DIS

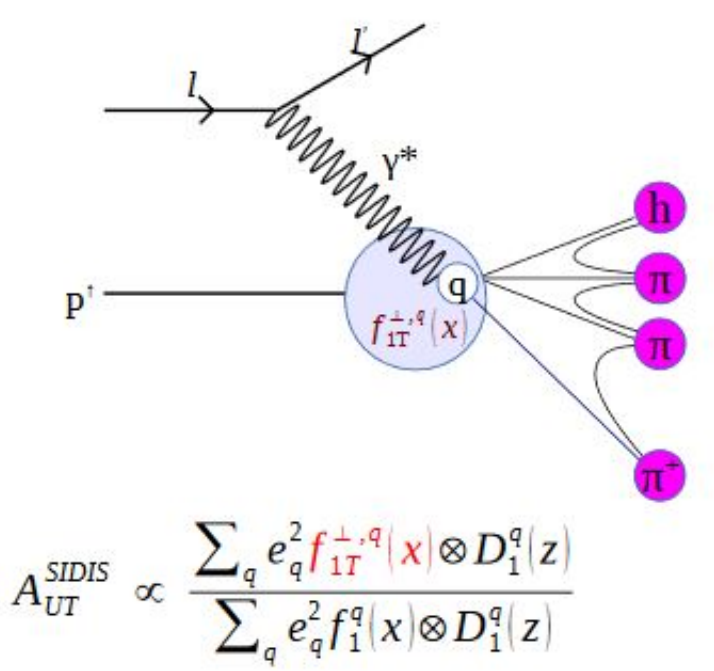

- L-R asymmetry in hadron production

- Sivers function convoluted with fragmentation function - not a clean measurement

$$
p+p^{\uparrow} \rightarrow \mu^{+} \mu^{-} X
$$

\section{Polarized Drell-Yan}

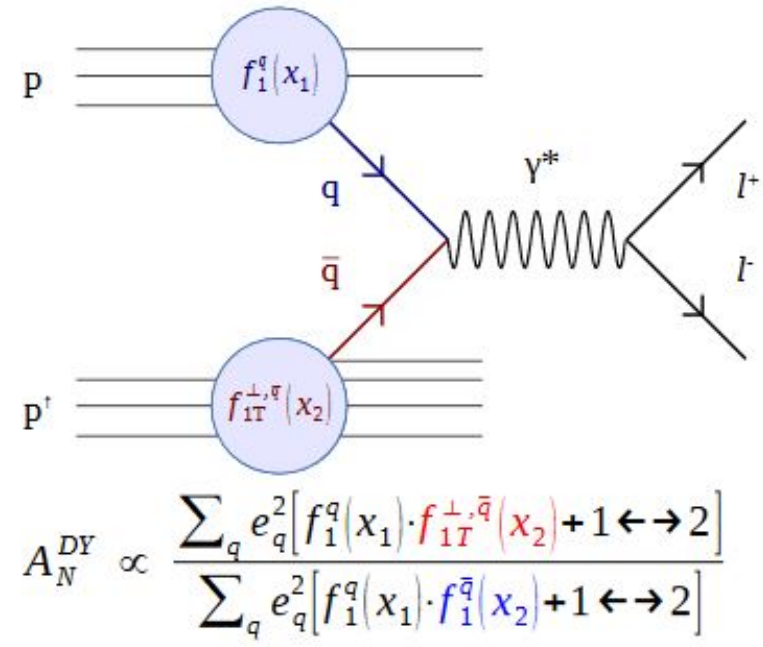
without fragmentation function - clean measurement 


\section{Spin Physics and E1039 (SpinQuest)}

SpinQuest purposes direct measurement sea-quark Sivers function using Drell-Yan productions from unpolarized $120 \mathrm{GeV}$ proton beam on a transversely polarized proton (neutron) target.

$$
e+p^{\uparrow} \rightarrow e^{\prime} \pi X
$$

Polarized Semi-Inclusive DIS

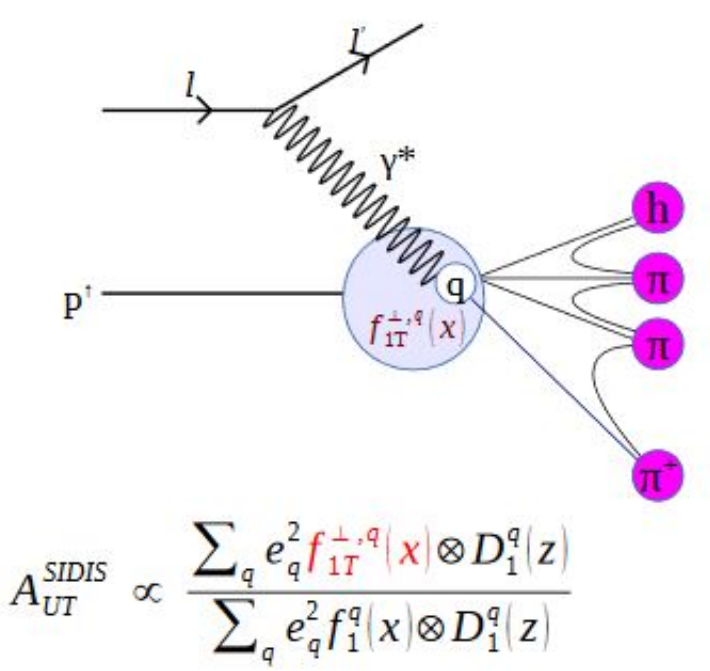

- L-R asymmetry in hadron production

- Sivers function convoluted with fragmentation function - not a clean measurement

$$
p+p^{\uparrow} \rightarrow \mu^{+} \mu^{-} X
$$

Polarized Drell-Yan

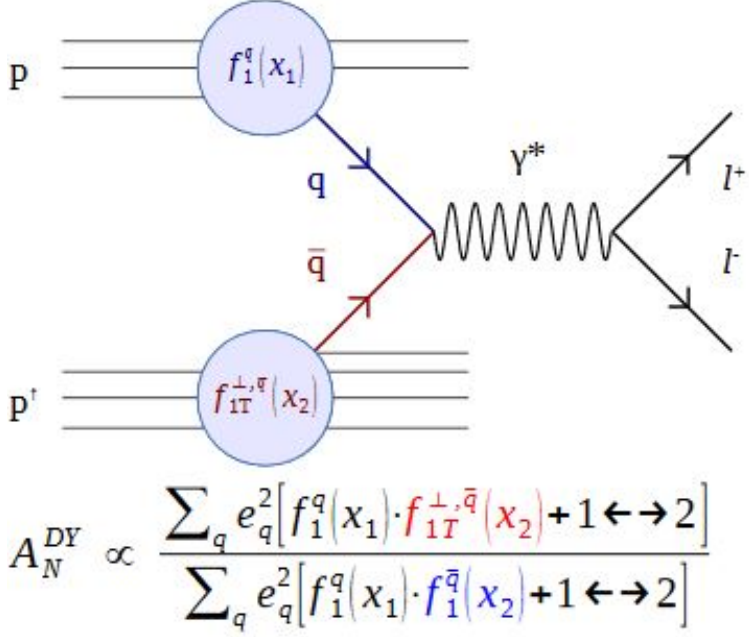

- L-R asymmetry in Drell-Yan production

- Direct measurement of Sivers function without fragmentation function - clean measurement 


\section{Target Inserts and Microwave Horn}

- Two proton inserts: One with four target cups (large), one with three target cups (small). As well as one deuteron insert with at three cold NMR coils.

- Proton inserts surrounded by carbon fiber shell for minimal thermal conductivity and guidance.

- Each insert has three thermal sensors to monitor the annealing plate temperatures.

- NMR coils installed on each target cup.
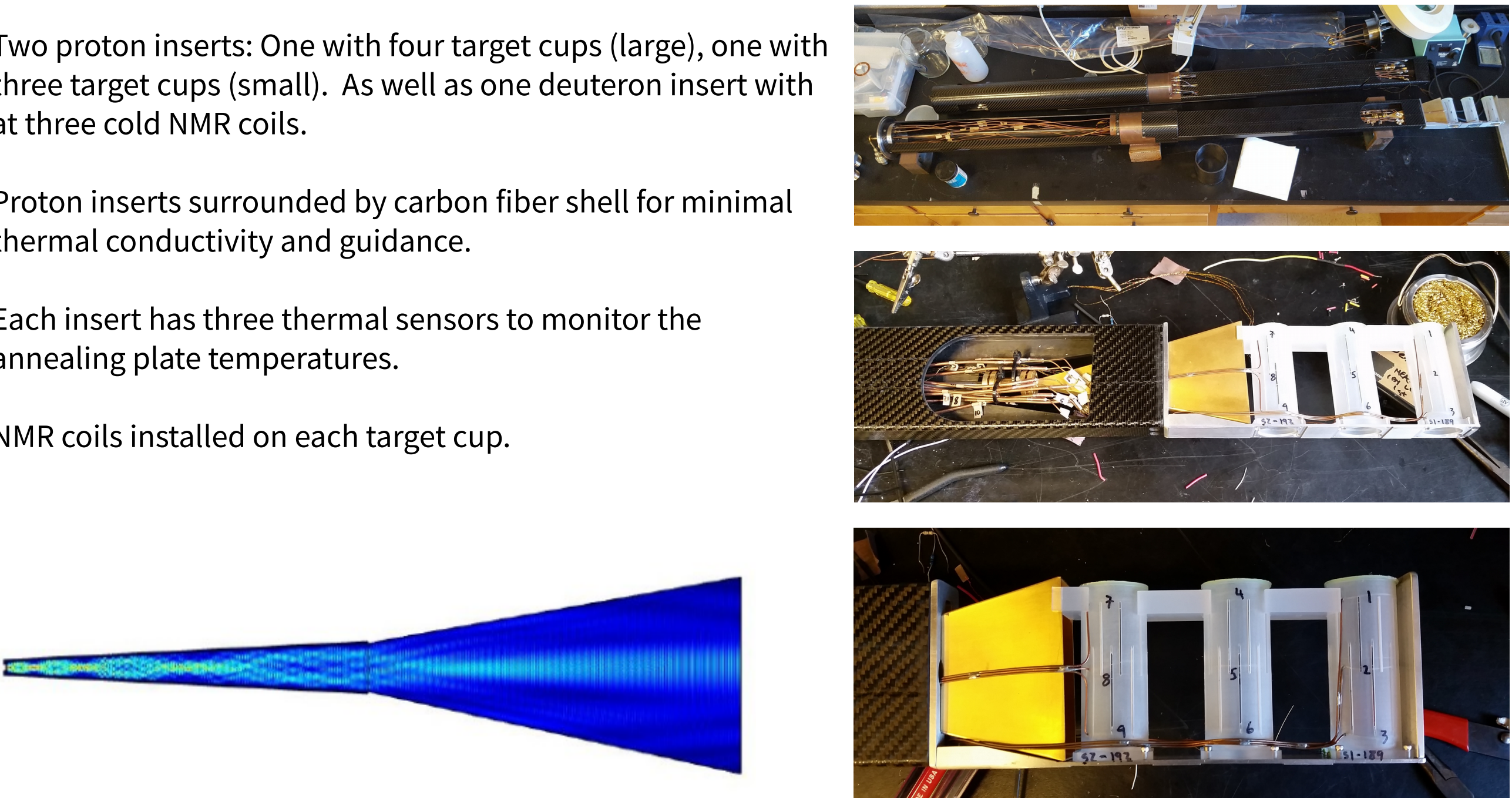


\section{Target Inserts and Microwave Horn}

- Two proton inserts: One with four target cups (large), one with three target cups (small). As well as one deuteron insert with at three cold NMR coils.

- Proton inserts surrounded by carbon fiber shell for minimal thermal conductivity and guidance.

- Each insert has three thermal sensors to monitor the annealing plate temperatures.

- NMR coils installed on each target cup.
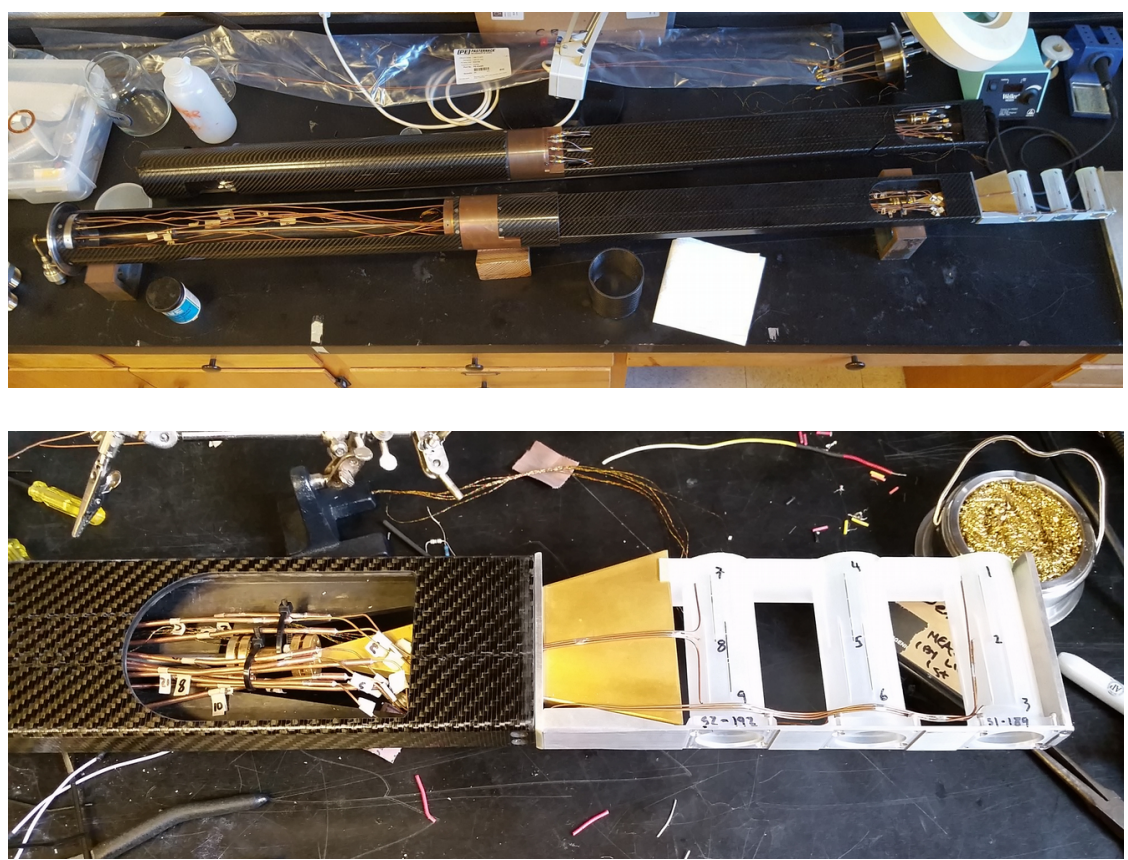

Cups for the proton and deuteron are filled with $\mathrm{NH} 3$ and ND3 respectively.

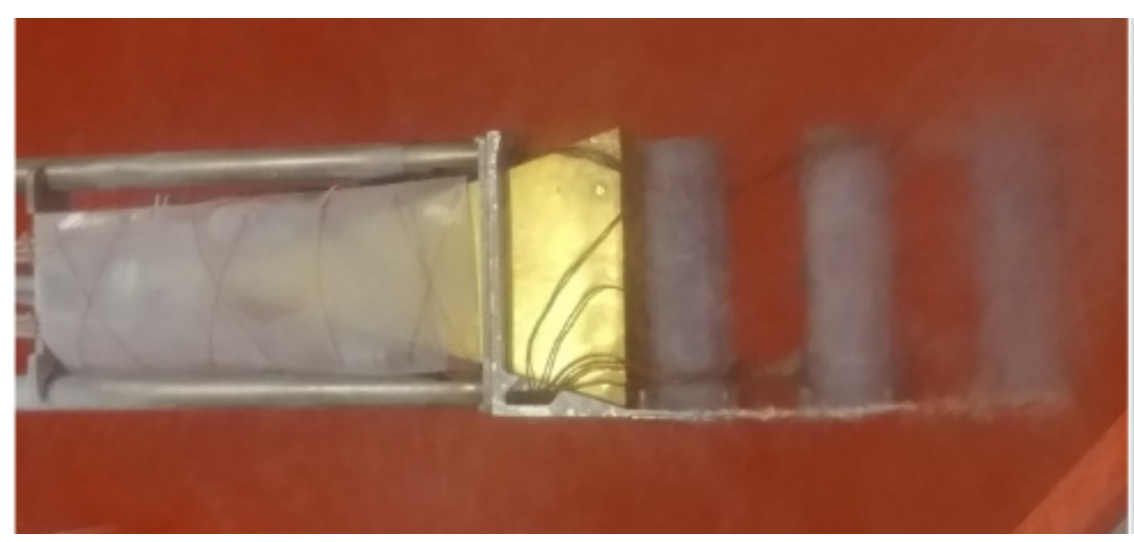

